\title{
Helio- and Asteroseismic Analysis Methods
}

\author{
Jesper Schou \\ Stanford University, HEPL Annex A201, Stanford, CA 94305-4085, \\ $U S A$
}

\begin{abstract}
Over the past couple of decades increasingly sophisticated analysis algorithms have been developed in helioseismology. Here I will describe some of these with an emphasis on those most relevant to asteroseismology and some of the lessons learned. I will also discuss some of the properties of stellar oscillations and how those affect the analysis.
\end{abstract}

\section{Overview}

Helioseismic analysis methods have become increasingly complex as the quality of the data, our understanding of the physics and the available computing power have increased. In section 2 I will briefly review some of these methods.

With the increase in quantity and quality of asteroseismic data (see reviews by Kurtz and Kjeldsen in these proceedings), it is natural to investigate how the helioseismic experience can be applied to the asteroseismic case and where new techniques need to be developed. I will briefly review this in Section 3.

\section{Helioseismic Data Analysis}

The analysis techniques are generally classified as either global or local. The former are effectively defined as those treating the oscillations as a sum of normal modes. I will review the typical steps in the global methods in the following subsections. For more details see Schou (1992) or Hill et al. (1996). The local methods are reviewed by Haber and Hill in these proceedings.

\subsection{Spherical Harmonic Transform}

The first step is to isolate the individual spherical harmonics. This is generally done by mapping the images to heliographic coordinates, multiplying by the spherical harmonic and integrating the result. However, only part of the solar surface is observed, and thus the spherical harmonics will not be perfectly isolated, causing so-called leaks. While those can be calculated, imperfections in our knowledge of the image geometry or mode physics will lead to errors.

\subsection{Temporal Fourier Transform}

The next step is to Fourier transform the time series of each spherical harmonic coefficient. While this is simple, a number of problems exist. In particular it is necessary to remove bad points and to prevent data gaps from causing spectral 


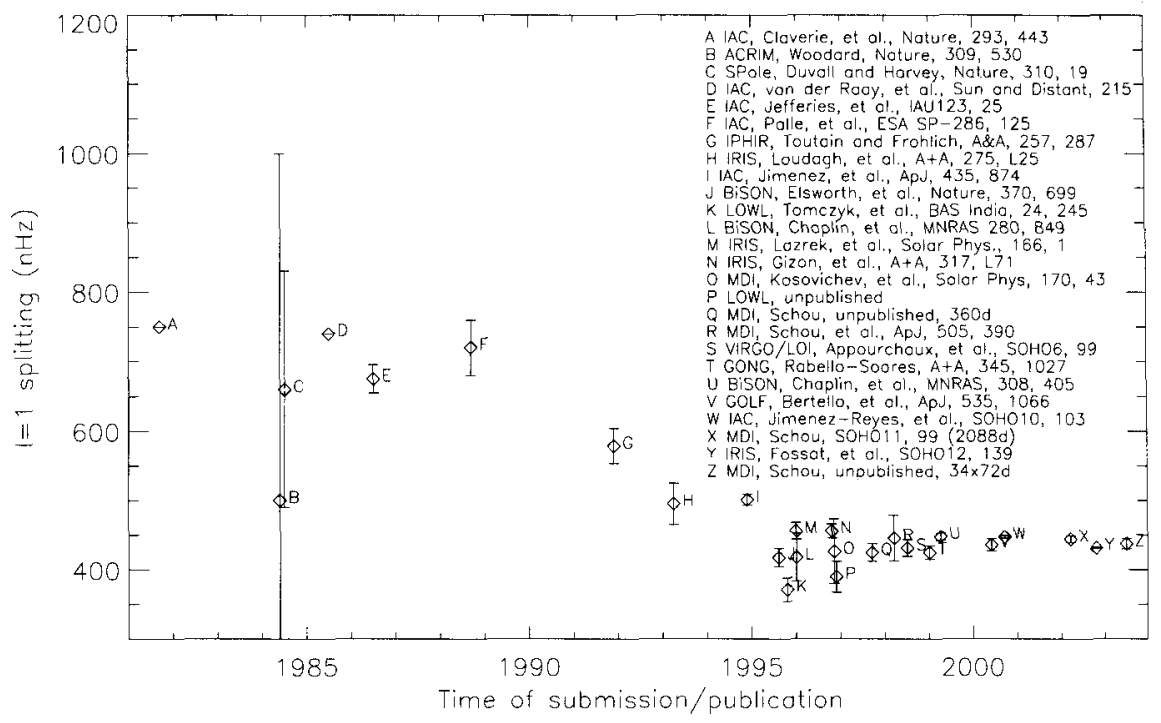

Figure 1. Splittings for $\ell=1$ (averaged over the available $n$ ) as a function of time of submission/publication/generation. Each data point is identified by the experiment and a reference is given.

artifacts. This is typically done by a combination of detrending and gap filling. Due to the large dynamic range in the spectra, it is essential to do this carefully.

\subsection{Parameter Estimation}

Finally the mode parameters need to be estimated from the spectra. This is, by far, the most complicated part of the analysis. Depending on the modes being studied the methods are categorized as either peakbagging or ridge fitting.

Peakbagging Here, modes with different spherical harmonic degree $\ell$ can be separated in the spectra, while the azimuthal orders $m$ may or may not be separated. The methods differ in which effects are taken into account and which parameters are fitted versus modeled. Some of the differences are illustrated by the algorithms used by the MDI and GONG projects (see Schou et al. 2002 and Basu et al. 2003 for a more detailed comparison).

The GONG method (Anderson et al. 1990; Hill et al. 1996) fits each $(n, l, m)$ ( $n$ is the radial order) separately; the fit is done in the power spectra, no leakage matrix is used and the parameters from a fit of a mode is not used to constrain the properties of that same mode when it appears as a leak.

The MDI method (Schou 1992) is more complex. All $m$ values at each $(n, l)$ are fitted simultaneously, a leakage matrix is used, the leak parameters are constrained and the fit is done in the Fourier domain. However, while using a leakage matrix and constraining the leak parameters should improve the fits, inaccurate leaks can affect the fitted parameters.

Ridge Fitting As leak spacings decrease and line widths increase, modes merge into ridges. While fitting those is fairly easy, it is difficult to relate the fitted 
parameters to those of the underlying modes, as this requires an accurate leakage matrix. For details see Rhodes et al. (2001) and Korzennik et al. (2004).

\section{Asteroseismic Data Analysis}

While parts of the asteroseismic analysis techniques, such as the Fourier transforms and peakbagging are similar to those used in helioseismology, other aspects, detailed below, are quite different. For a review see Appourchaux (2003).

\subsection{Time series problems}

Among the challenges here are low $\mathrm{S} / \mathrm{N}$, short time series, low duty cycle, uneven data quality and uneven sampling when combining data from different sites. An example of the problems encountered is shown in Figure 1. The systematic trend in the $\ell=1$ splitting is most likely not solar in origin, rather it is probably caused by a bias in the estimates for short time-series of spatially unresolved data, such as those available early on for the Sun and those currently available for other stars. While this bias is fairly well understood Figure 1 does illustrate that it is essential to understand the properties of the analysis methods.

\subsection{Mode Identification Difficulties}

Another problem is to identify the modes. For the Sun the basic parameters are well known, modes can be observed to high $\ell$, the spectrum is fairly simple and the identities thus easy to determine. For other stars this is not the case, as illustrated in Figure 2, which shows the tentative identifications of peaks in a WIRE spectrum of $\alpha$ Cen A originally reported by Schou and Buzasi (2001) and the identifications based on additional data (Bouchy \& Carrier 2002).

Another set of problems is caused by the complex behavior of the frequencies in some stars caused by the simultaneous presence of modes of different character (e.g. $\mathrm{p}, \mathrm{f}$, and $\mathrm{g}$ modes). The different modes may have very different line widths or amplitudes and some modes may not be excited to a measurable amplitude or be invisible due to geometric effects. Rapid rotation can also lead to nonlinearities and even more confusion.

\subsection{Miscellaneous}

Other problems encountered include such things as large inherent frequency variations and confusion or frequency changes caused by binary stars. Also a number of decisions have to be made, such as deciding on the number of stars observed versus duration and how the HR diagram should be covered.

\section{Conclusion}

While some of the above may sound discouraging, the situation is actually quite encouraging. Several ground-based experiments are delivering data, WIRE and MOST have been or are operating in space and other projects, such as COROT, Kepler and Eddington are in various stages of planning or under construction.

Regarding the data analysis it is important to understand the physics, the instrument and the data analysis methods. Hopefully the lessons learned in 

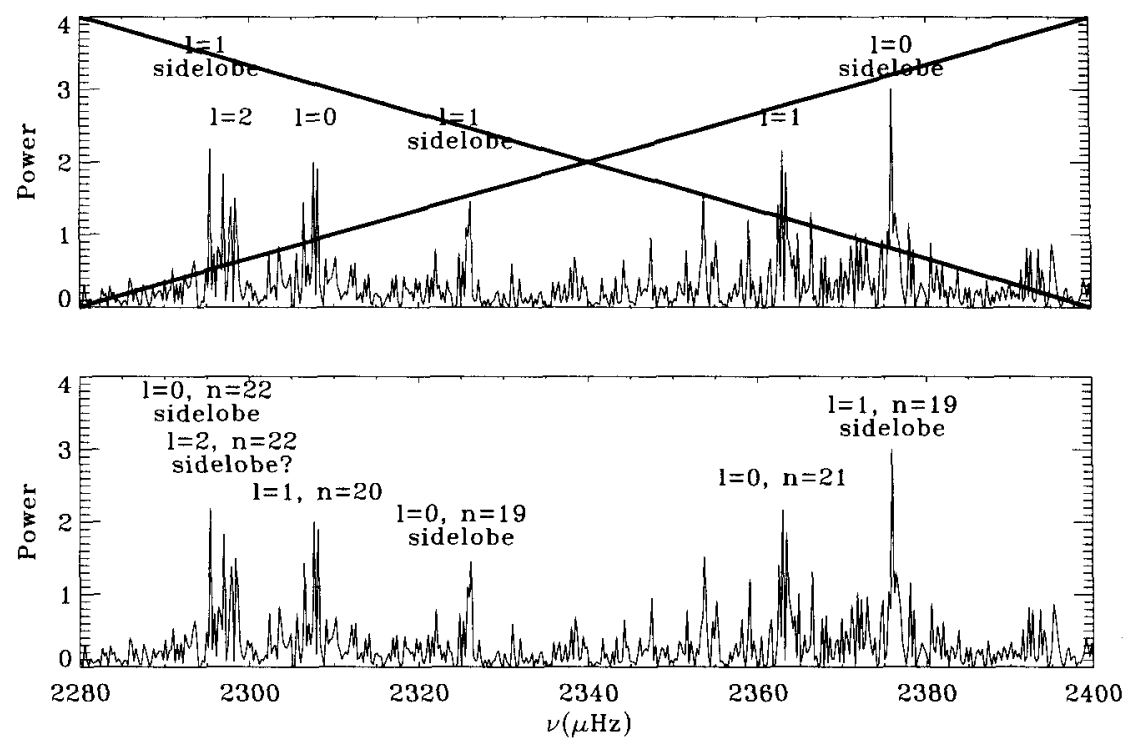

Figure 2. Spectra of $\alpha$ Cen A as observed by WIRE (Schou \& Buzasi 2001). Top: original mode identifications. Bottom: revised.

helioseismology will be valuable for asteroseismology and allow for rapid progress to be made.

Acknowledgments. This work was supported by NASA through grants NAG5-10483 and contract NAS5-02139.

\section{References}

Anderson, E. R., Duvall, Jr., T. L. \& Jefferies, S. M. 1990, ApJ, 364, 699

Appourchaux, T. 2003, Ap\&SS, 284, 109

Basu, S., et al. 2003, ApJ, 591, 432

Bouchy, F. \& Carrier, F. 2002, A\&A, 390, 205

Hill, F., et al. 1996, Science, 272, 1292

Korzennik, S. G., Rabello-Soares, M. C. \& Schou, J. 2003, ApJ, 602, 481 (stro-ph/0207371)

Rhodes, E. J., Jr, et al. 2001, ApJ, 561, 1127

Schou, J. 1992, PhD Dissertation, University of Aarhus, Denmark

Schou, J. 1998, SOHO 6/GONG 98, ESA SP-418, 47

Schou, J. \& Buzasi, D. L. 2001, SOHO 10/GONG 2000, ESA SP-464, 391

Schou, J., et al. 2002, ApJ, 567, 1234 